\title{
Pharmacokinetic/pharmacodynamic (PK/PD) evaluation of Caspofungin in a population of critically ill septic patients
}

Chiara Adembri*, Gianluca Villa*, Beatrice Loriga*, Andrea Novelli**, Elia Rosi**, Lorenzo Tofani***

*University of Florence, Department of Health Sciences, Section of Anesthesiology and Intensive Care, University of Florence, Italy

**University of Florence, Department of Health Sciences, Section of Pharmacology, University of Florence, Italy

***University of Florence, Department of Neurosciences, Psycology, Drug Research and Child Health, University of Florence, Italy.

Careggi Hospital, Florence, Italy

\section{Background and Goal of the study}

Major alterations in the PK/PD of antimicrobial drugs are well documented in critically ill septic patients, but little data are available for echinocandins ${ }^{(1,2)}$. Echinocandins represent the first therapeutic choice for septic patients admitted to Intensive Care Units (ICU) when an invasive candida infection is suspected or proven.

The aims of the present study were the following: $i$ ) to describe the alterations of PK of the first commercially available echinocandin, Caspofungin, in critically ill septic patients and ii) to carry out a PK/PD analysis in order to assess whether optimal targets are reached in these patients.

\section{Materials and Methods}

Both surgical and medical ICU patients receiving Caspofungin as empirical/targeted therapy were enrolled if they were aged $\geq 18$ years and diagnosed with severe sepsis or septic shock. Demographic, clinical and biochemical parameters were collected. Seven plasma samples (before the first dose and after 1, 2, 4, 8, 12 and 24h) and four urine samples (from 0-6, 6-12, $12-18,18-24 \mathrm{~h}$ intervals) were taken following the first dose of Caspofungin.

Plasma and urine concentrations were measured using a validated High Performance Liquid Chromatography (HPLC) method. PK/PD analysis was performed using SAS 9.3 software.

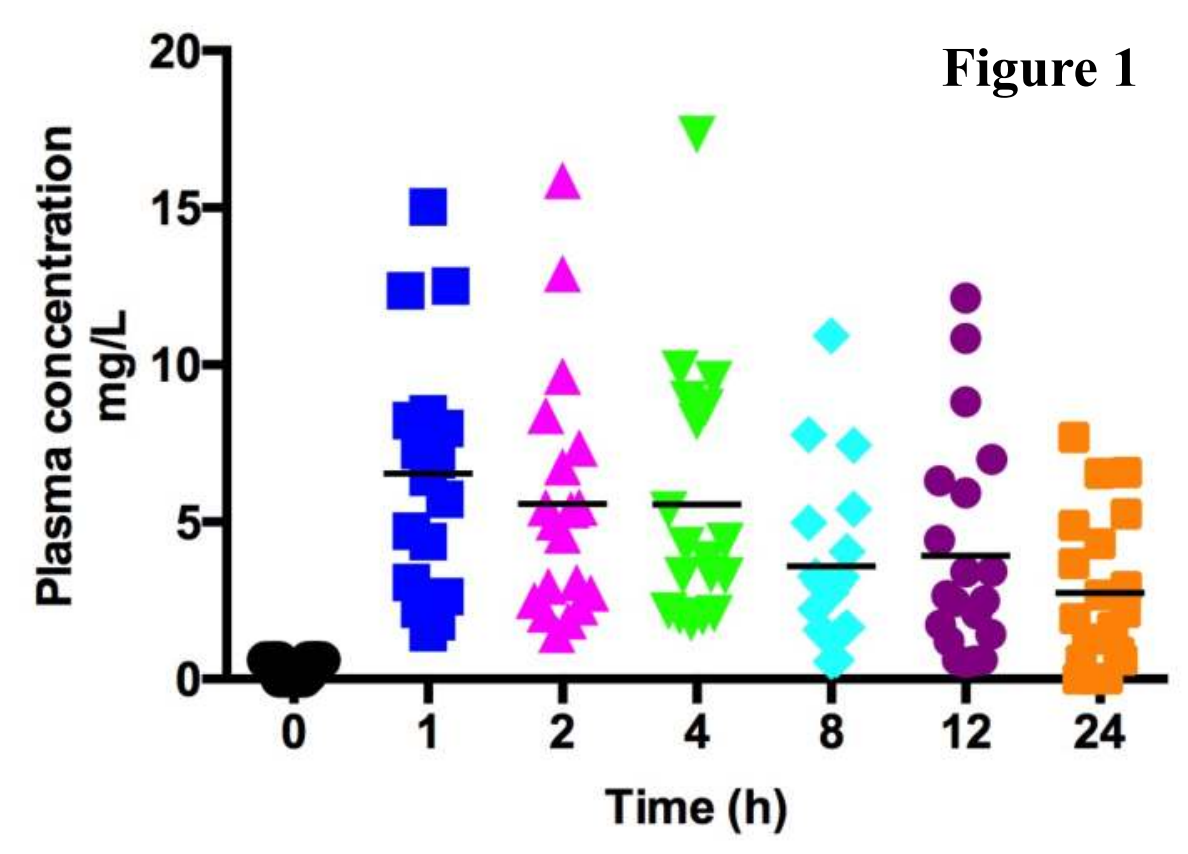

\begin{tabular}{|c|c|c|c|}
\hline Table 2 & All patients $(\mathrm{n}=20)$ & Group A (n=9) & Group B (n=11) \\
\hline $\mathrm{C}_{\max }(\mathrm{mg} / \mathrm{L})$ & $9.9 \pm 9.4$ & $* 4.4 \pm 2.4$ & $* 14.4 \pm 10.6$ \\
\hline $\mathrm{C}_{\min }(\mathrm{mg} / \mathrm{L})$ & $2.5 \pm 2.5$ & $* * 0.7 \pm 0.7$ & $* * 4.1 \pm 2.4$ \\
\hline $\mathrm{AUC}_{0-24}(\mathrm{mg} / \mathrm{h} / \mathrm{L})$ & $104.3 \pm 81.0$ & $* * 43.9 \pm 14.7$ & $* * 153.8 \pm 79.5$ \\
\hline Urinary recovery (\%) & $9.4 \pm 11.4$ & $11.0 \pm 15.4$ & $8.0 \pm 6.8$ \\
\hline $\mathrm{C}_{\max } / \mathrm{MIC}$ & $39.6 \pm 37.5$ & $* 17.5 \pm 9.5$ & $* 57.8 \pm 42.3$ \\
\hline $\mathrm{AUC} / \mathrm{MIC}$ & $417.4 \pm 324.2$ & $* * 175.4 \pm 58.7$ & $* * 615.3 \pm 318.1$ \\
\hline
\end{tabular}

All data are expressed as mean values $\pm \mathrm{SD}$.

$\mathrm{AUC}=$ area under the plasma concentration-time curve. MIC according to the CLSI breakpoint

* $\mathrm{p}<0.05$, group A vs group B; ** $\mathrm{p}<0.01$, group A vs group B

\section{Results and Discussion}

Twenty consecutive patients entered the study. Ten patients had been admitted for medical reasons and the remaining ten for surgery. Details of the clinical and demographic parameters are reported in Table 1. Plasma concentration after the first dose is reported in Figure 1.

The main PK data are reported in Table 2.

According to the PK analysis, a high inter-individual variability occurred and patients could be divided into 2 significantly different groups, A and B. The profile of the plasma concentration time curves in the 2 groups is as depicted in Figure 2, panels $\mathrm{A}$ and $\mathrm{B}$ and $\mathrm{C}$ ).

The main $\mathrm{PK} / \mathrm{PD}$ index, expressed as mean $\mathrm{AUC} / \mathrm{MIC}$ ratio, $\mathrm{Cmax} / \mathrm{MIC}$ and the urinary recovery are as reported in Table 2. Cmac/MIC and AUC/MIC were significantly higher in group $B$ than in group A (ANOVA, $\mathrm{p}<0.01$ ) Patients showed higher mean urinary recovery of the un-metabolized molecule when compared to healthy volunteers: $9.4 \%$ versus $2-3 \%$, with no difference between group A and B.
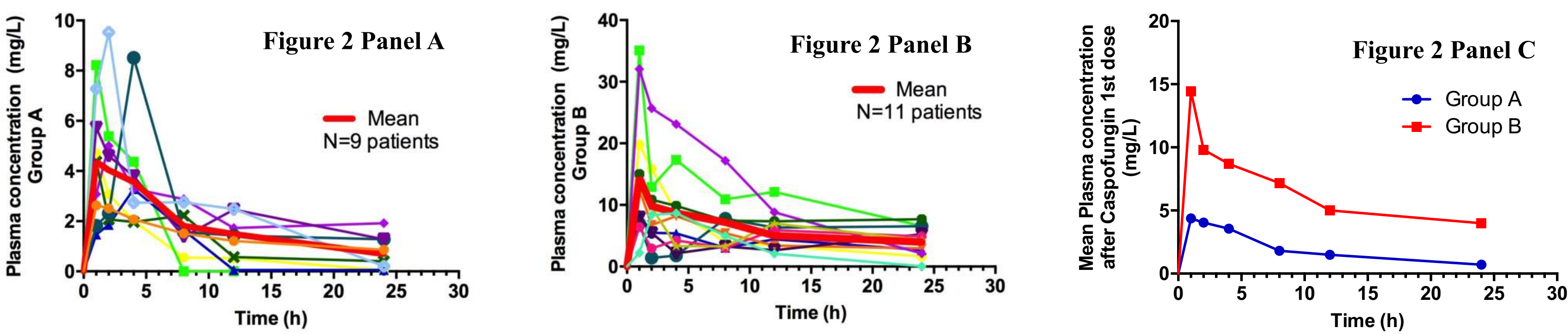

\section{Conclusions}

High inter-individual variability was observed for Caspofungin PK in the critically ill septic patients, with some of them having significant lower plasma concentrations and increased urinary recovery compared to healthy volunteers. This phenomenon affects the probability to achieve optimal PK/PD targets in some ICU patients and suggests that TDM for Caspofungin should be performed in the ICU setting.

1. Udy AA, Roberts JA, Lipman J., Clinical implications of antibiotic pharmacokinetic principles in the critically ill., Intensive Care Med. 2013 Dec;39(12):2070-82

2. Stone JA, Holland SD, Wickersham PJ, Sterrett A, Schwartz M, Bonfiglio C, Hesney M, Winchell GA, Deutsch PJ, Greenberg H, Hunt TL, Waldman SA. 2002. Single- and multiple- dose pharmacokinetics of caspofungin in healthy men. Antimicrob Agents Chemother 392 46:739-45. 